\title{
Graves' disease, celiac disease and liver function abnormalities in a patient - clinical manifestation and diagnostic difficulties
}

\author{
Magdalena Góra-Gębka1凶, Małgorzata Woźniak², Joanna Cielecka-Kuszyk , Maria Korpal- \\ Szczyrska4, Katarzyna Sznurkowska1', Maciej Zagierski', Irena Jankowska², Katarzyna Plata- \\ Nazar ${ }^{1}$, Barbara Kamińska1 and Anna Liberek ${ }^{5}$
}

\begin{abstract}
1Department of Pediatrics, Pediatric Gastroenterology, Hepatology and Nutrition, Medical University of Gdansk, Gdańsk, Poland; ${ }^{2}$ Department of Gastroenterology, Hepatology and Eating Disorders Children's Memorial Hospital in Warsaw, Warsaw, Poland; ${ }^{3}$ Department of Patology, Children's Memorial Hospital in Warsaw, Warsaw, Poland; ${ }^{\circ}$ Department of Pediatrics, Hematology, Oncology and Endocrinology, Medical University of Gdansk, Gdańsk, Poland; '5Faculty of Health Sciences with Subfaculty of Nursing Medical University of Gdansk, Gdańsk, Poland
\end{abstract}

\begin{abstract}
Autoimmune diseases due to probable common pathogenesis tend to coexist in some patients. Complex clinical presentation with diverse timing of particular symptoms and sophisticated treatment with numerous side effects, may cause diagnostic difficulties, especially in children. The paper presents diagnostic difficulties and pitfalls in a child with Graves' disease, celiac disease and liver function abnormalities.
\end{abstract}

Key words: autoimmune disease, Graves' disease, celiac disease, liver abnormalities, children

Received: 13 October, 2013; revised: 12 March, 2014; accepted: 06 April, 2014; available on-line: 06 June, 2014

\section{INTRODUCTION}

Graves' disease is a common autoimmune disease with a prevalence of $1 / 1000$ in children (Jacobson et al., 1997). Autoimmune diseases such as Graves' disease, celiac disease and autoimmune hepatitis, due to probable common etiopathogenesis tend to coexist in some patients (Meloni et al., 2001; Strassburger et al., 2002).

Complex clinical presentation with diverse timing of occurrence of particular symptoms and treatment with numerous side effects, may cause diagnostic difficulties. Liver function abnormalities are common in this group of patients. Children appear to be a special group of patients with immature immune system, altered pharmacokinetics of some drugs and compliance.

The aim of this paper is to present diagnostic difficulties and pitfalls in a child with Graves' disease, celiac disease and liver function abnormalities.

\section{CASE REPORT}

A 12-year-old girl with no evident burden in perinatal and familial medical history is presented. At the age of 8 the diagnosis of Graves' hyperthyroidism was established — with thyroid hormone fT4 $50 \mathrm{pmol} / \mathrm{l}$, undetectable thyroid stimulating hormone (TSH), anti-thyroid peroxidase (TPO) antibodies - $598 \mathrm{IU} / \mathrm{ml}$, anti-thyroglobulin (TG) antibodies $1837 \mathrm{IU} / \mathrm{ml}$, and no nodules in the ultrasound scan of thyroid gland. Screening for celiac disease was negative by then and decreased IgA level-0, $16 \mathrm{~g} / 1$ was noted. Initially, the patients was treated with methimazol (no consent for surgical therapy was ob- tained), and next, because of an ophtalmopathy the steroids were introduced in a primary full dose of $40 \mathrm{mg}$ $(1 \mathrm{mg} / \mathrm{kg} / \mathrm{d})$ for 6 weeks, and then in the reduced doses for 4 months. Although the therapy brought some improvement, full remission was not achieved - every attempt to withdraw from methimazol was unsuccessful. Still, no consent for surgical therapy was obtained. After 3 years propylthiouracyl (PTU) and Levothyroxine sodium were introduced.

At the age of 12 the girl was transferred to our department from Department of Pediatric Endocrinology because of liver injury with cholestasis. In medical history infection of respiratory tract with cephalosporins therapy prior to the occurrence of jaundice was noted. On admission the girl was jaundiced, with enlargement of thyroid gland, exophthalmos and hepatosplenomegaly. Laboratory tests showed increased transaminases activity (AlAT $977 \mathrm{U} / 1$, Aspat $629 \mathrm{U} / \mathrm{l}$ ), bilirubin levels (2, $3 \mathrm{mg} / \mathrm{dl}$, direct bilirubin $1,7 \mathrm{mg} / \mathrm{dl}$ ), increased activity of gamma-glutamyl transpeptidase (GGTP $120 \mathrm{U} / \mathrm{l}$ ) and bile acids levels (179 micrmol/l). The full blood count, acute phase proteins, sedimentation rate, protein pattern, coagulation and thyroid function tests were within normal ranges; merely elevated immunoglobulin $G$ level (IgG 17, $43 \mathrm{~g} / \mathrm{l}$ ), and slightly decreased immunoglobulin A $(\operatorname{IgA}-0,2 \mathrm{~g} / \mathrm{l})$ were noted. The ultrasound scans showed enlarged liver with no features of cholestasis, no lymphadenopathy. In the further investigations the most common infectious (HBV, HCV, HAV, CMV, E-BV, HSV-1, HSV-2, Toxoplasma gondi) and metabolic (alfa1-antitripsin deficiency, Wilson's disease) diseases were excluded. Immunological test were negative for antiliver-kidney microsomal (LKM-1), anti-smooth muscle (SMA), anti-nuclear (ANA), anti-neutrophil cytoplasmatic (ANCA), bile ductless (BDA) anibodies next to the test for anti-transglutaminase (tTG) and anti-endomysium (IgG-EMA) antybodies. Due to normal thyroid gland

e-mail: mgora@gumed.edu.pl

Abbreviations: AILD, autoimmune liver disease; $\mathrm{AlH}$, autoimmune hepatitis; PSC, primary slerosing cholangitis; ANA, anti-nuclear antibododies; ANCA, anti-neutrophil cytoplasmatic antibodies; AIAT, alanine aminotransferase; Aspat, aspartate aminotransferase; BDA, bile ductless antibodies; $C D$, celiac disease; CMV, cytomegalovirus; EMA, antiendomysium antibodies; E-BV, Ebstein-Barr virus; fT4, free thyroxine 4; GGTP, gamma-glutamyl transpeptidase; HAV, hepatitis A virus; $\mathrm{HBV}$, hepatitis $B$ virus; $\mathrm{HCV}$, hepatitis $C$ virus; $\mathrm{HSV}$, herpes simplex virus; IgA, immunoglobulin $A$; LKM, anti-liver-kidney microsomal antibodies; PTU, propylthiouracyl; SMA, anti-smooth muscle antibodies; TSH, thyroid stimulating hormone; TPO, thyroid peroxidase; TG, thyroglobulin; tTG, anti-transglutaminase antibodies 
A

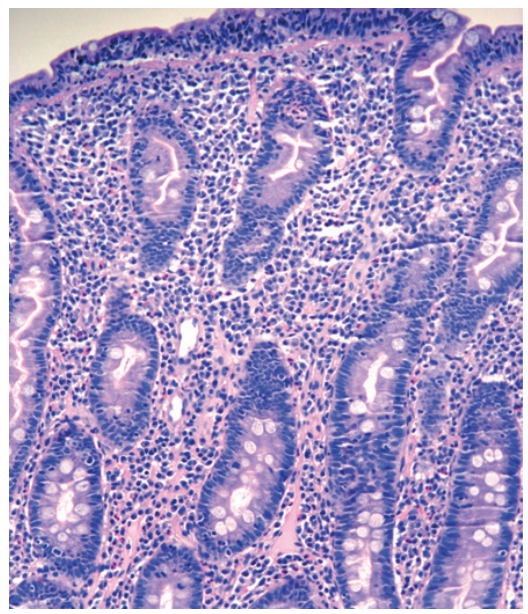

B

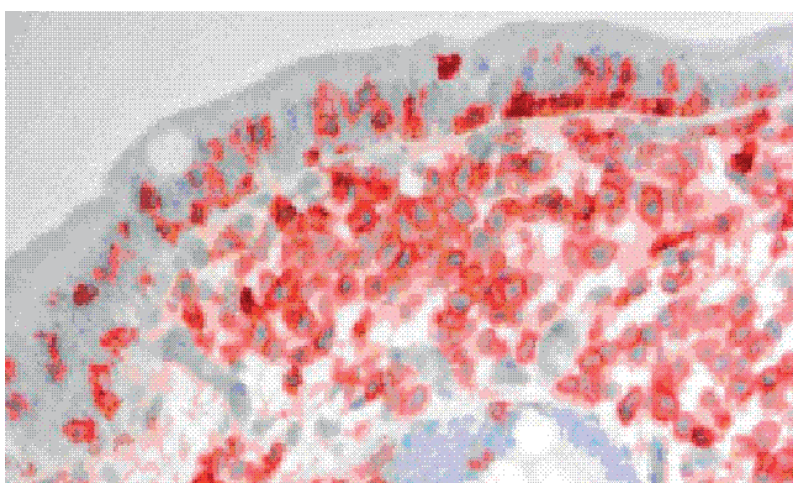

Figure 1. Duodenal biopsy no 91848: (A) Villous atrophy with crypt hyperplasia (H\&E); (B) Increased number of intraepithelial lymphocytes (Immunohistochemical stain with CD3).

function test and possible risk of side effects, PTU was withdrawn and conservative hepatoprotective therapy (ursodeoxycholic acid, ornithine, essential fosfolipids, dextrose infusions) were introduced to the cure, which resulted in the fast liver function tests normalization.

In 2 months time the patient was admitted again to the hospital, because of the hyperthyroidism (fT4 35 $\mathrm{pmol} / \mathrm{l}$ ) and elevated transaminases activity (AlAT up to $300 \mathrm{U} / 1$ with no cholestasis in laboratory tests). Laboratory tests showed no features of infectious disease. Tests for the autoimmune processes showed the presence of anti-smooth muscle (SMA, 1:640) and anti-nuclear (ANA; 1:320) antibodies as well as the presence of anti-transglutaminase (tTG) and anti-endomysium (IgGEMA) antybodies. The diagnosis of celiac disease was confirmed by the histopathological changes within the intestinal mucosa - grade III in Marsh scale (Fig. 1) — vilious atrophy, crypt hyperplasia, increased density of intraepithelial $\gamma / \delta$ T-cells (Marsh, 1992). After the initial treatment with thyrostatics (methimazol) and gluten free diet, strumectomy (accompanied with the liver biopsy) was performed. Liver histopathological findings showed inflammatory (grading 2 in the Batts' scale) and fibrotic (staging 2) changes with no evident features of autoimmune process such as portal bridging necrosis and fibrosis with massive inflammatory infiltrates of lymphocytes and plasma cells and interface hepatitis (Fig. 2) (Batts et al., 1995). Consecutive substitutive hormonal therapy

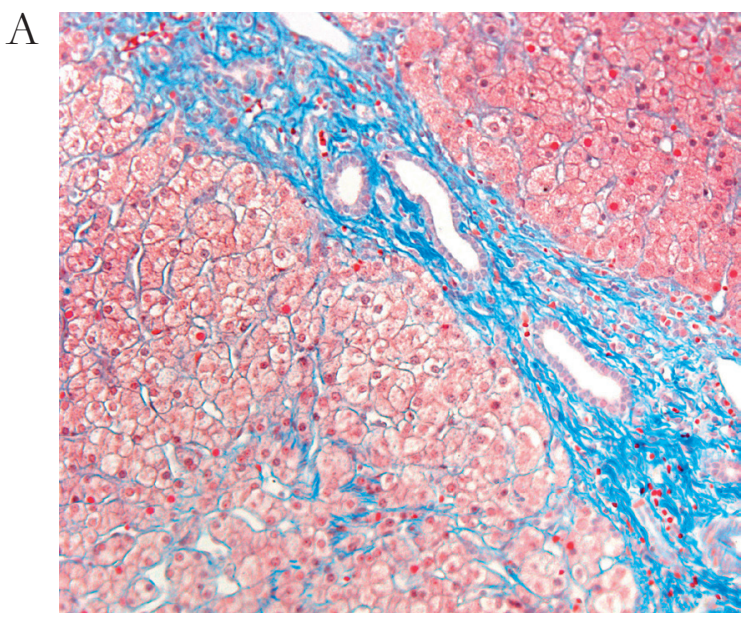

and gluten free diet finally resulted in euthyreosis, normal liver function tests. Control liver biopsy performed in 4 months time showed full regression of inflammatory changes and minor fibrous changes (staging S0/S1) (Fig. 3).

\section{DISCUSSION}

Liver function abnormalities were noted few times in the course of the Graves' disease in our patient. Laboratory abnormalities ranged from the increase in aminotransferases activity with cholestasis in the time of PTU treatment, euthyrodism and respiratory tract infection treated with antibiotics to high activity of liver enzymes next to hyperthyroidism and tissue antibodies presence strongly suggestive of some other autoimmune diseases. Sophisticated and comprehensive diagnostic approach was warranted along with side effects of some drugs, which had to be taken under consideration.

Thyrotoxicosis stands for one of the potential causes of liver dysfunction in patients with Graves' disease. The combination of increased oxygen consumption and inadequate perfusion leading to hepatocyte hypoxia is regarded as the main pathogenic mechanism of liver damage in these patients (Shimizu, 2008). However, in the presented case the timing of liver function abnormalities was poorly correlated with high levels of thyroxin.

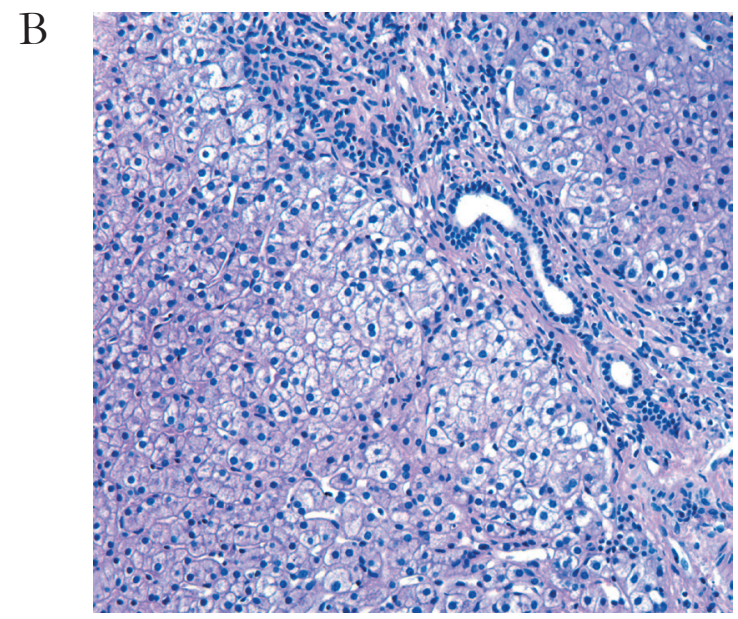

Figure 2. Liver biopsy (I) no 90643: (A) Portal tract with fibrous septa (Azan stain), (B) Portal tract with mild inflammation and fibrosis (H\&E). 


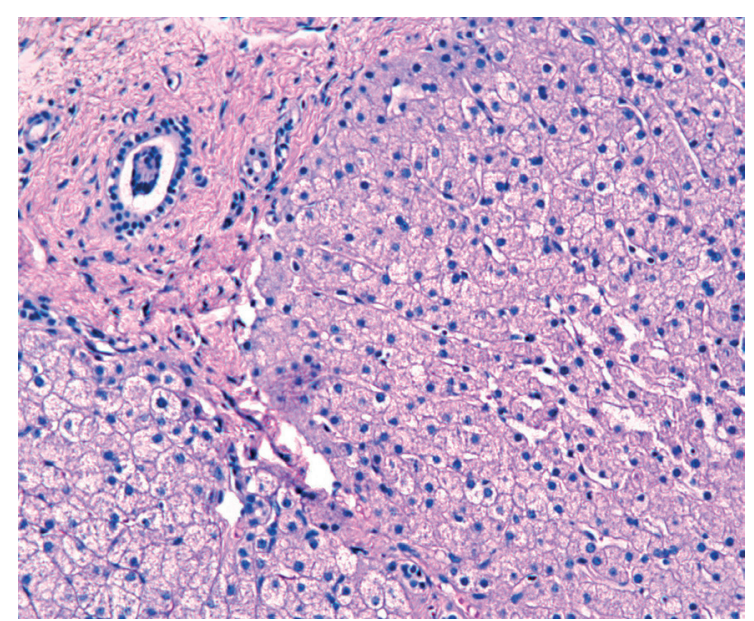

Figure 3. Control liver biopsy after 4 months (II) no. 91207: Portal tract is enlarged with fibrosis without inflammatory infiltrates (H\&E).

Methimazol and propylthiouracyl with some side effects, like increase in liver enzymes, are the antithyroid drugs commonly used in Graves' disease. Most cases of PTU-induced hepatic injury occur in the first few months of PTU therapy (Lazar et al., 2000; Kim et al., 2001). The PTU-induced hepatic injury is manifested histologically as acute cholestatic, hepatocellular or mixed forms. In pediatric patients adverse reactions related to PTU therapy, including moderate increase of liver function tests, are noted in $71 \%$ of pre-pubertal vs. 25\% of post-pubertal children (Lazar et al., 2000; Kim et al., 2001). Among children receiving transplants due to drug-induced acute liver failure, PTU appears to be the third cause following acetaminophenon and isoniasid (Russo et al., 2004). Clinical manifestation, the results of laboratory tests, the histology of the liver tissue, and the effect of PTU withdrawal are strongly suggestive of this mechanism of liver injury, which was noted at the time of euthyrodism in our patient.

There is a high frequency of anti-neutrophil cytoplasmatic (ANCA) antibodies in patients with Graves' disease treated with PTU. However, in few cases only, a classical ANCA associated vasculitis with kidney or liver manifestation (type 1 autoimmune hepatitis) is observed (Targan et al., 1995; Cui et al., 2003; Sato et al., 2004). Little is still known about some other antibodies in this clinical entity, which may or may not have clinical significance. No ANCA was detected in our patient who also presented with normal kidney function. The presence of SMA and ANA was noted in our patient and appeared to be suggestive of the autoimmune process like autoimmune hepatitis type 1 . Autoimmune hepatitis type 2, with the presence of LKM antibodies is reported to appear more often in either children with $\mathrm{AIH}$ or patients with Graves' disease (Targan et al., 1995; Cui et al., 2003; Sato et al., 2004). According to the autoimmune hepatitis score system, our patient did not fulfilled the score criteria for the definite diagnosis of AIH. The liver histopathological findings showed no evident features of autoimmune process like portal bridging necrosis and fibrosis with per-portal inflammatory infiltrate of lymphocytes, plasma cells and neutrophils. No glucocorticoids was administered prior to the liver biopsy, which could have abolished the characteristic lymphoplasmacytic infiltrate typical for AIH.
Three types of liver disease are described in celiac disease: mild, transient liver dysfunction, chronic liver disease (with severe fibrosis especially in patients with $\mathrm{CD}$ and $\mathrm{HCV}$ infection) and autoimmune liver disease (AILD) (Davidson, 2002; Freeman, 2006). They may be within the spectrum of the clinical manifestation of the same disease, where congenital factors and gluten exposure may influence the pattern of liver dysfunction, or may reflect liver abnormalities of different pathogenesis. Non-specific hepatitis with moderate increase in aminotransferases activity is a quite common clinical entity in celiac disease (CD) (Lindberg et al., 1978; Maggiore et al., 1994).

In most cases, aminotransferase normalize with 12 months of gluten-free diet (Novacek et al., 1999; Bardella et al., 1999). Histological changes are mild and include Kupffer cell hyperplasia, mononuclear cell infiltration, steatosis and mild fibrosis (Novacek et al., 1999; Bardella et al., 1999). The latter finding was probably the one, which was noted in the control biopsy in our patient.

In the course of disease and treatment our patient presented with liver injury with the presence of SMA and ANA, but also with the features of cholestasis, which may be suggestive of either autoimmune hepatitis, primary slerosing cholangitis (PSC) and autoimmune slerosing cholangitis (AISC). LKM appear to be the most frequently noted autoantibodies in $\mathrm{AIH}$ in the course of either CD or Graves' disease (Volta et al., 1998; Davidson et al., 2002; Cui et al., 2003). However, in some recent studies AIH type 1 is reported to coexist more often with $\mathrm{CD}$ even in pediatric group of patients (Rubio-Tapaia et al., 2007; Diamanti et al., 2008). Although according to the autoimmune hepatitis score system, we should have been suspicious of AIH in our patient, the liver histopathological findings showed no typical features of AIH, like portal bridging necrosis and fibrosis with peri-portal inflammatory infiltrate of lymphocytes, plasma cells and neutrophils. Some studies report the prevalence of PSC in CD patients to be increased 4-fold to 8-fold than in individuals without CD (Rubio-Tapaia et al., 2007; Ludvigsson et al., 2007). In other, the prevalence of CD is $1.6 \%$ in the group of PSC patients (Volta et al., 1997; Rubio-Tapaia et al., 2007). Liver histology showed no typical features of cholestasis and lymphocytic portal inflammatory process with increased intra-epithelial lymphocytes in biliary ductal epithelium (Frejman et al., 1994; Freeman, 2006).

In summary we highlight the wide range of probable mechanisms involved in the liver injury in the course of Graves' disease and CD. The diagnosis of autoimmune liver disease may cause some difficulties and some other pathogenetic mechanisms should be taken under consideration.

Conflicts of interest and source of funding: none declared.

\section{REFERENCES}

Bardella MT, Vecchi M, Conte D, Del Nino E, Fraquelli M, Pacchetti S, Minola E, Landoni M, Cesana BM, De Franchis R(1999) Chronic unexplained hypertransaminaseaemia may be caused by the occult celiac disease. Hepatology 29: 654-657.

Batts KP, Ludwig J (1995) Chronic Hepatitis. An Update on Terminology and Reporting. Am J Surg Pathol 19: 1409-1417.

Cui B, Abe M, Hidata S Nakanishi S, Matsuura B, Michitaka K, Yamamoto K, Horiike N, Onji M (2003) Autoimmune hepatitis associated with Graves' disease. Intern Med 42: 331-333.

Davidson S (2002) Coeliac disease and liver dysfunction. Arch Dis Child 87: 293-296. 
Diamanti A, Basso MS, Pietrobatista A, Nobili V (2008) Prevalence of celiac disease in children with autoimmune hepatitis. Dig Liv Dis 40: 965.

Freeman HJ (2006) Hepatobiliary and pancreatic disorders in celiac disease. World J Gastroenterol 12: 1503-1508.

Frejman HJ, Kwan WC (1994) Occult celiac disease associated with lymphocytic sclerosing cholangitis. Can J Gastroenterol 8: 249-252.

Jacobson DL, Gange SJ, Rose NR, Graham NM.(1997) Epidemiology and estimated population burden of selected autoimmune diseases in the United States. Clin Immunol Immunopathol 84: 223-243.

Kim HJ, Kim BH, Han YS Yang I, Kim KJ, Dong SH, Kim HJ, Chang YW, Lee JI, Chang R (2001) The incidence and clinical characteristics of symptomatic propylthiouracyl-induced hepatic injury in patients with hyperthyroidism: a single-center retrospective study. Am J Gastroenterol 96: 165-169.

Lazar L, Kalter-Leibovici O, Pertzelan A, Weintrob N, Josefsberg Z, Phillip M (2000) Thyrotoxicosis in prepubertal children compared to with pubertal and postpubertal patients, J Clin Endocrinol Metab 85: $3678-3682$

Lindberg T, Berg NO, Borulf S, Jacobson I.(1978) Liver damage in coeliac disease or other food intolerance in childhood. Lancet 1 : 390-391.

Ludvigsson JF, Elfstrom P, Broome U, Ekbom A, Montgomery SM.(2007) Celiac disease and risk of liver disease: a general population-based study. Clin Gastroenterol Hepatol 5: 63-69.

Maggiore G, Ceccarelli M, Colombo Giacomo C De, Virgilis S De, Musumeci S, Ventura A (1994) 141 Hepatic lesions in childhood celiac disease: a multicentric retrospective study. I Pediatr Gastroenterol Nutr 19: 691-696.

Marsh M (1002) Gluten, major histocompatibility complex and the small intestine. A molecular and immunobiologic approach to the spectrum o gluten sensitivity('celiac sprue'). Gastroenterology 102: 330_ 354.

Meloni GF, Thomasi PA, Bertoncelli A Fanciulli G, Delitala G, Meloni $T$ (2001) Prevalence of silent celiac disease in patients with autoimmune thyroiditis from Northern Sardinia. I Endocrinol Invest 24: 298-302.

Novacek G, Miehsler W, Wrba F, Ferenci, P, Penner E., Vogelsang H (1999) Prevalence and clinical importance of hypertransaminaseaemia in celiac disease. Eur J Gastroenterol Hepatol 11: 283-288.

Rubio-Tapaia A, Murray J (2007) The liver in Celiac Disease. Hepatology 46: $1650-1658$

Russo MW, Galanko JA, Shrestha R, Fried MW, Watkins P.(2004) Liver transplantation for acute liver failure from drug induced liver injury in the Unitated States. Liver Transplant 10: 1018-1023.

Sato H, Hattori M, Fujieda M, Sugihara S, Inomata H, Hoshi M, Miyamoto S (2000) High prevalence of antineutrophil cytoplasmatic antibody in childhood onset Graves' disease treatment with propyltiouracil. J Cin Endocrinol Metab 85: 4270-4273.

Shimizu Y (2008). Liver in systemic disease World J Gastroenterol 14: 4111-4119.

Strassburg CP, Manns MP( 2002) Autoantibodies and autoantigens in autoimmune hepatitis. Sem Liver Dis 22: 339-352.

Targan SR, Landers C, Vidrich A, Czaja AJ (1995) High-titer antineutrophil cytoplasmatic antibodies in type-1 autoimmune hepatitis. Gastroenterology 108: 1159-1166.

Volta U, De Franceschi L, Molinaro N, Cassani F, Muratori L, Lenzi M, Bianchi FB, Czaja AJ (1998) Frequency and significance of antigliadin and anti-endomysial antibodies in autoimmune hepatitis. Dig Dis Sci 43: 2190-2195. 